\title{
Effet des androgènes sur l'activité sportive
}

\author{
G. Peres \\ Laboratoire de Physiologie et de Médecine du Sport, CHU Pitié-Salpétrière, \\ 94 bd de l'Hôpital, 75013 Paris
}

\section{RESUME}

Les sécrétions androgéniques semblent apporter un bénéfice à la performance sportive en :

- renforçant la motivation, la volonté, la résistance au stress, voire l'agressivité, et en augmentant la résistance à la fatigue générale perçue, d'où la possibilité d'accroître les charges d'entraînement,

- renforçant probablement la trame et la minéralisation, et donc la résistance mécanique, osseuses en intervenant sur les métabolismes protéiques et calciques,

- stimulant, en relation avec l'érythropoïétine, l'érythropoièse au niveau de la moelle hématogène,

- augmentant la glycémie, mais aux dépens d'une diminution de la tolérance au glucose,

- stimulant la mobilisation des triglycérides du tissu adipeux,

- participant à mieux gérer, chez le sportif entraîné, les réserves de glycogène musculaire dont dépend l'endurance maximale aérobie et à les économiser par une utilisation accrue, par le métabolisme oxydatif au niveau des mitochondries, des triglicérides provenant du tissu adipeux. Le transport membranaire du glucose est modifié,
- augmentant la masse protéique musculaire, en stimulant l'incorporation des acides aminés en vue de la synthèse protéique, et par un effet antagoniste des glucocorticoïdes en diminuant la dégradation protéique. La force musculaire est augmentée sous entraînement. Ces effets anabolisants ne sont pas retrouvés au niveau des tendons.

- et en modulant les défenses immunitaires, renforcées ou amoindries selon la dose physiologique ou pharmacologique d'androgènes.

Mots-clés : Androgènes, physiologie, exercice musculaire, sport, performance.

L'utilisation de diverses aides physiques et chimiques pour améliorer la performance remonte à la plus haute antiquité. Ainsi les grecs anciens consommaient des graines de sésame, les indiens des Andes mâchaient des feuilles de coca et les aborigènes d'Australie des plantes locales pour leurs effets stimulants et anti-fatiguants. Les romains consommaient des testicules d'animaux pubères à des fins d'augmentation de la force et de la masse musculaire.

Les androgènes ont des effets démontrés très nombreux sur les organes et fonctions. Quelques-uns intéressent le domaine des performances sportives.

A côté des effets psycho-physiologiques, les androgènes semblent avoir une action sur 
la morphologie même du cerveau. Ainsi le nucleus des régions pré optiques est plus volumineux chez les rats mâles [7] et la portion moyenne du noyau de l'amygdale médiane est également plus élevée [20]. Le ganglion sympathique cervical supérieur est plus grand [18]. La testostérone est capable d'augmenter le facteur de croissance nerveuse [3] conduisant à une augmentation de la survie neuronale [10]. Ces actions paraissent s'expliquer par une concentration élevée de récepteurs aux androgènes dans l'hypophyse, l'hypothalamus, la région préoptique, le septum et l'amygdale [18].

Il semble que les androgènes sont responsables d'un appétit supérieur, d'une activité plus grande chez les rats que les rattes [35].

La testostérone est capable d'augmenter les sécrétions et les taux plasmatiques de l'hormone de croissance et de la somatomédine C $[17,25]$. Cette action passe par des peptides opiacés endogènes [23].

Il a pu être démontré que les androgènes stimulent sélectivement des neurones du système somato-moteur et les circuits associés à l'agression (cité in 3 ). Des récepteurs aux androgènes sont localisés sur les motoneurones alpha et y jouent un rôle de régulation de leur longueur chez l'adulte [16].

Ces effets centraux et périphériques sont peut-être les principales raisons d'augmentation de la force chez le sportif sous apports d'androgènes ou simplement en termes de comparaison entre hommes et femmes.

Les effets comportementaux des androgènes sont de type effet stimulant sur le système nerveux central comme par exemple la vivacité mentale, l'amélioration de l'humeur, l'augmentation de la mémoire, de la concentration, de l'agressivité, du comportement d'opposition, de l'euphorie. La motivation et la volonté sont renforcées et la sensation de fatigue diminuée. Tout ceci bénéficie à la réussite dans les sports d'opposition ou pour ceux qui impliquent un exercice épuisant. Sous apport pharmacologique de dérivés de testostérone il a pu être démontré que le profil EEG est proche de celui obtenu avec les psycho-stimulants [3].

A l'aide de différents types de questionnaires chez l'adolescent il a pu être démontré une relation significative entre la testostéronémie et le tonus émotionnel, l'agressivité et le besoin de dominance $[21,27,28]$. La diminution de la tolérance à la frustration semble également dépendre de la testostérone. Chez l'adulte l'application de questionnaires d'anxiété, de dépression ou de tests de personnalité ou d'hostilité [30] ont montré une corrélation entre le débit de production de testostérone, mesuré par la méthode de perfusion constante, et la somme des réponses d'hostilité [22].

Les relations entre la sécrétion de testostérone et un certain nombre de comportements ou de qualités psychomotrices ne sont cependant pas bien établies ou même sont l'objet de discussion. Il en est ainsi du temps de réaction, de l'estimation des temps, de la sensation de confort physique, des affects positifs : ils semblent se détériorer avec l'augmentation des niveaux de testostéronémie [12].

Une santé mentale positive, une motivation pour obtenir des résultats supérieurs, pour se battre plus ou pour terminer plus fortement, sont corrélées positivement avec la testostéronémie [29].

Il semble exister une corrélation positive entre le niveau de testostéronémie et le niveau de stress psychologique sans que puissent être bien précisés les liens entre l'un et l'autre et en particulier si une testostéronémie élevée était un facteur de meilleure résistance au stress ou non; c'est plutôt le niveau de stress qui serait plus particulièrement déterminant du niveau de testostéronémie [15].

Il a été plusieurs fois rapporté et cela paraît assez communément admis actuellement 
que la relation entre testostéronémie et résistance à la fatigue était élevée. Le maintien pendant la saison d'une testostéronémie élevée permet une bonne résistance à la fatigue et donc autorise des entraînements plus longs, plus fréquents ou plus intenses avec des délais de récupération plus rapides et donc une répétition plus élevée des entraînements [6]. Cette résistance à la fatigue s'accompagne souvent d'une irritabilité et d'une agressivité supérieures.

$\mathrm{Au}$ niveau morphologique, les androgènes sont stimulant de la croissance chez l'adolescent, action qui paraît s'exercer en synergie avec l'hormone de croissance et les somatomédines [25]. Mais on sait que cette accélération de croissance est rapidement arrêtée par la soudure précoce des cartilages de conjugaison. Une diminution de la calciurie et une augmentation de la minéralisation osseuse conduisent à une ossification accentuée. Augmentation de la surface ostéoïde, augmentation de la surface des ostéoblastes mais aussi des ostéoclastes aboutissent à une apposition minérale osseuse et à une vitesse de formation de l'os supérieures chez le mâle par rapport à la femelle [32, 33].

La composition corporelle est modifiée par la testostérone, diminution de la masse grasse avec diminution de l'épaisseur des plis cutanés, augmentation de la masse maigre mise en évidence par une augmentation de la circonférence de l'avant-bras et de la cuisse et une diminution de l'épaisseur des plis cutanés.

Sur le tissu adipeux de fait les androgènes semblent avoir un effet sur la mobilisation différenciée selon qu'il s'agit de réserves lipidiques sous-cutanées ou intra-abdominales.

La lipolyse accentuée par les androgènes relève d'une activation des $\beta$-adrénorécepteurs et de l'adényl-cyclase.

La proportion d'eau corporelle augmente $d u$ fait d'une rétention [8].
Cette modification de la composition corporelle s'accompagne d'une augmentation de la dépense d'énergie quotidienne.

$\mathrm{Au}$ niveau sanguin une augmentation du taux d'hémoglobine, du nombre de globules rouges et en conséquence de l'hématocrite a été décrite du fait des androgènes, permettant de prédire une augmentation du VO2max significative [13]. L'hémostase est modifiée avec augmentation de la coagulabilité du sang sans que l'on sache actuellement très précisément quels en sont les mécanismes [2].

L'augmentation de l'érythropoïèse relève d'une stimulation du métabolisme de la moelle osseuse hématogène. Elle est facilitée par l'induction de la synthèse de l'hémoglobine, action en synergie avec l'érythropoïétine. La stimulation des ARN est le support de cette augmentation de la synthèse protéique.

Un effet hyperglycémiant a été décrit depuis longtemps. Il se traduit par une diminution de la tolérance au glucose et un hyperinsulinisme.

Les effets des androgènes sur les HDL et LDL cholestérol sont assez bien décrits : augmentation des LDL et VLDL, diminution HDL cholestérol. Il s'agit là plutôt d'effets secondaires néfastes.

Sur le système immunitaire à la fois des effets de suppression immunitaire lors d'apports chroniques de testostérone et de stimulation des thymocytes ont été décrits ; en fait lorsqu'il s'agit d'imprégnation physiologique avec stimulation des cellules du thymus, de la moelle osseuse, de la rate et des nodules lymphatiques avec sécrétion de facteur immuno-modulateur. A l'état physiologique donc la testostérone aurait plutôt un effet potentialisateur de l'immunité (cité in 14).

Au niveau myocardique et musculaire, mais pas au niveau hépatique, la testostérone a un effet d'épargne du glycogène pendant l'exercice sous-maximal. 
Au niveau du muscle la testostérone semble avoir un effet modulateur sur le métabolisme du glycogène. Ceci était démontré dans les muscles squelettiques de rats entraînés en endurance. La concentration en glycogène de muscles de rats sédentaires n'est pas modifiée sous testostérone. A l'entraînement dans le soleus mais pas dans l'EDL le contenu en gycogène est augmenté par l'entraînement, expliqué par une diminution de l'activité de la glycogène phosphorylase active. Chez les rats entraînés la testostérone provoque une augmentation du contenu en glycogène par une augmentation de l'activité de la glycogène synthétase et une diminution concomitante de l'activité de la glycogène phosphorylase active, tant dans l'EDL que dans le soleus [4].

Sous l'effet de la testostérone, la captation du glucose est augmentée au niveau de la cellule musculaire, elle ne l'est pas au niveau du foie. C'est d'une des explications à l'absence d'effet de la testostérone sur le glycogène hépatique.

La testostérone chez les animaux entraînés occasionne un déplacement de la distribution des fibres musculaires vers des fibres plus oxydatives et ceci dans les deux muscles étudiés [4].

Les stéroïdes anabolisants modifient les enzymes mitochondriales et sarcotubulaires participant à la synthèse protéique dans le muscle squelettique de rats [26].

Les androgènes ont effet anticatabolique protéique réduisant globalement la dégradation des protéines musculaires. Ceci est particulièrement sensible lorsque sévit une atrophie musculaire d'origine cortisolique [11]. Cette action paraît liée à une fixation croisée sur les récepteurs aux glucocorticoïdes avec les stéroïdes anabolisants.

La testostérone a un effet trophique musculaire sous forme d'hypertrophie des fibres individuelles sans changer leur nombre, donc sans hyperplasie $[1,19]$. Il faut noter que se sont les muscles de la ceinture sca- pulaire, du thorax et des épaules, qui sont plus sensibles aux androgènes que ceux de la ceinture pelvienne et des membres inférieurs [36].

Un point essentiel c'est la nécessité de l'exercice d'intensité élevée ou bien de phénomènes dégénératifs ou de lésions musculaires pour que soit observé un effet anabolisant des androgènes sur le muscle squelettique [31].

La synthèse protéique est stimulée par la testostérone endogène ou les stéroïdes anabolisants exogènes à la fois au niveau transcriptionnel et translationnel et ceci parce qu'il existe des récepteurs à haute affinité pour la testostérone dans le muscle [34].

Il en résulte une augmentation de la masse maigre, de la force musculaire et de la performance sportive et ceci plus particulièrement chez les sujets déjà entraînés qui augmentent encore leur quantité d'entraînement [5, 9], soulignant bien les effets sur la tolérance au volume d'entraînement, sur la récupération ou la fatigue.

Rappelons que sur la force les androgènes passent aussi par une amélioration du recrutement central.

Au niveau cardiaque il existe une augmentation du transport des hexoses et des acides aminés et du flux calcique transmembranaire, ce qui se traduit par une performance cardiaque qui semblerait augmentée sous testostérone. Les effets sur les mitochondries, les myofibrilles ainsi que sur les pressions artérielles et sur la contractilité myocardique paraissent moins intéressants si ce n'est délétères chez le sportif.

En conclusion : on peut affirmer que la testostérone participe très largement à la réussite sportive avec des effets significatifs tout particulièrement au niveau du système nerveux central et au niveau de la fibre musculaire, intéressant tant les sportifs d'endurance que de force. 
Ces effets doivent cependant être considérés dans le contexte des effets secondaires de ces hormones d'une part et de leur statut pendant l'exercice, diminution significative sous forte charge d'entraînement, d'autre part, traités par ailleurs.

\section{REFERENCES}

1. ALWAY SE, STARKWEATHER LA. : Effect of anabolic steroids on new fiber formation and fiber area during stretch-overload. J Appl Physiol, 1993, 74 : 832-837.

2. ANSELI JE, TIARKS C, FAIRCHILD CK : Coagulation Abnormalities Associated with the Use of Anabolic Steroid. Am Heart J, 193, 125 : 367-371.

3. BAHRKE M.S, YESALIS C.E. WRIGHT J.E. : Psychological and Behavioural Effects of Endogenous Testosterone Levels and Anabolic-Androgenic Steroids Among Males. Sports Med, 1990, 10 : 303337

4. BREDA V.E, HANS A, KEIZER A,GEURTEN P, KRANENBURG V.G, MENHEERE P.P.C.A., KUIPERS H, GLATZ JAN.F.C. : Modulation of glycogen metabolism of rat skeletal muscles by endurance training and testosterone treatment. Pflùgers Arch, 1993, $424:$ 294-300.

5. DEHENIN L. : Androgènes et dopage : La prise abusive d'androgènes est analysée en fonction du mode d'action anabolisante, de l'influence sur la force musculaire et des conséquences endocriniennes. Repr hum horm 1992, 5 ; 619-631.

6. FREED DL, BANKS AJ, LONGSON D, et al. : Anabolic steroids in athletics : crossover double-blind trial on weightlifters. British Med J, 1975, $2: 471$ 473.

7. GORSKI RA, GORDON JH, SHRYNE JE. : Evidence for a morphological sex difference within the medial preoptic area of the rat brain. Brain research, 1978, 148 : 333-346.

8. GREGORY J.W., GREEN S.A, THOMPSON J., SCRIMGEOUR C.M., RENNIE M.J. : Effects of oral testosterone undecanoate on growth, body composition, strength and energy expenditure of adolescent boys. Clin Endocrinol, 1992, 37 : 207-213.

9. HAUPT H.A; ROVERE G.D. : Anabolic steroids: A review of litterature. Am. J. Sports Med. 1984,12,469-484.

10. HENDRY IA, CAMPBELL. : Morphometric analysis of rat superior cervical ganglion after axotomy and NGF treatment. J Neurocytol, 1976, 5 : 351360 .
11. HICKSON R.C, CZERWINSKI S.M, FALTUDO M.T, YOUNG A.P. : Glucocorticoid antagonism by exercise and androgenic-anabolic steroids. Med. Sci. Sport Exerc., 1990, $22: 331-340$.

12. HOUSER BB. : An investigation of the correlation between hormonal levels in males and mood, behavior and physical discomfort. Hormones Behav, $1979,12: 185-197$.

13. JAKOB E., FUCHS V, HOFFMANN R., KEUL J. : Physiological, Hormonal and Hematological Changes in strenuous physical Exercice under the influence of testosterone-enanthate. Med. klinik abt sport und leistungsmedizin. Freiburg FRG

14. KOCHARIAN C.D., WELDER A.A. : Anabolicandrogenic steroids : in cell culture. In Vitro Cell. Dev. Biol., 1993, 29A : 433-438.

15. KREUZ LE, ROSE RM, JENNINGS JR. : Suppression of plasma testosterone levels and psychological stress. Arch gen psychiatry, 1972, 26 : 479-482.

16. KURZ EM, SENGELAUB DR, ARNOLD AP. : Androgens regulate the dentrite length of mammalian motorneurons in adulthood. Science, 1986, 232, 395-398.

17. MARTIN LG CLARK JW, CONNOR TB. : Growth hormone secretion enhanced by androgens. Journal of Clinical Endocrinology, 1968, 38 : 425428.

18. McEWEN BS. : Binding and metabolism of sex steroids by the hypothalamic-pituitary unit : physiological implications. Annual Reviews Physiol , 1980, $42: 97-110$.

19. MOORADIAN A.D., MORLEY J.E., KORENMAN S.G. : Biological actions of androgens. Endocrine Rev, 1987, 8: 1-28.

20. NISHIZUKA M. ARAI Y. : Sexual dimorphism in synaptic organization in the amydala and its dependence on neonatal hormone environment. Brain Research, 1981, 212 : 31-38.

21. OLWEUS D., MATTSON A, SCHALLING D et al. : Testosterone, aggression, physical and personality dimensions in normal adolescent males. Psychosomatic Med, 1980, $42:$ 253-269.

22. PERSKY H, SMTTH KD. : Relation of psychologic measures of aggression and hostility to testosterone production in man. Psychosomatic Med. 1971, $33: 265-277$.

23. ROGOL AD, MARTHA PM, BLIZZARD RM. : Anabolic-androgenic steroids profoundly affect growth at puberty. In Erinoff \& Lin (eds) Anabolic steroid abuse, NIDA Research Monographn, National Institute on Drug Abuse, Rockville MD, 1990. 
24. ROME HP, BRACELAND FJ. : Psychological response to corticotropin, cortisone, and related corticoid substances. J Am Med Ass, 1952, 148 : 27-30.

25. ROSENFIELD RL, FURLANETTO R. : Physiologic testosterone or estradiol induction of puberty increases plasma somatomedin C. J Pediatrics, $1985,107: 415-417$.

26. SABORIDO A., VILA J., MOLANO F., MEGIAS A. : Effect of anabolic steroids on mitochondria and sarcotubular system of skeletal muscle. J. Appl. Physiol. 1991, 70 : 1038-1043.

27. SUSMAN EJ, INOFF-GERMAIN G., NO'TTELMAN ED, et al. : Hormones emotional dispositions, and aggressives attributes in young adolescents. Child Development, 1987, 58 : 1114-1134.

28. SUSMAN EJ NOTTELMAN ED, INOFF-GERMAIN G et al. : The relation of relative hormonal levels and physical development and social-emotional behavior in young adolescents. J Youth Adolescence, $1985,14: 245-264$.

29. TANAKA H, YAMAMOTO K, IMAMURA E, et al. : Relationship beween plasma testosterone levels and psychological mood states or aptitudes in sportsmen. Proc First intern olympic Comm world Congr Sports Sci, Colorodo Springs, 1989.

30. THURSTONE LL. : Thurstone Temperament Schedule : examiner's manual, Sci Research Associates, Chicago, 1950.

31. TINGUS S.J. CARLSEN R.C. : Effect of continuous infusion of an anabolic steroid on murine skeletal muscle. Med. sci sports exerc, 1993, 25 : 485-494.

32. VANDERSCHUEREN D., HERCK V.E., SUIKER A.MH. et al. : Bone and mineral metabolism in the androgen-resistant (testicular feminized) male rat. $\mathrm{J}$ bone mineral res, 1993, $8: 801-809$.

33. VANDERSCHUEREN D., BOUILLON R. : Androgens and bone. Calcif tissue Int, 1995, $56: 341$ 346.

34. VIRU A, KORGE P. : Role of anabolic steroids in the hormonal regulation of skeletal muscle adaptation. J Steroid Biochem, 1979,11 : 931-932.

35. WADE GB. : Sex hormones, regulating behaviors and body weight. Adv Behav 1976, $6: 201$.

36. WILSON J.D. : Androgen abuse by athletes. Endocrine Rev., 1988, $9:$ 181-199.

\section{ABSTRACT}

Androgenic effects on sport characteristics

G. Peres

Androgenic hormones seem to be of beneficial effects on sports performance :

- they increase motivation, will, aggressiveness, resistance to the stress and to the fatigue, leading to an increase of the training quantity,

- they increase bone mineralization and probably mechanical resistance,

- they stimulate the bone marrow and so, with the erythropoietin, the erythropoiesis,

- they increase the tendancy to hyperglycemia, but with a decrease of the tolerance to the glucose,

- they stimulate the fatty acids mobilization from the adipose tissue, for their utilization in the muscle during the exercise,

- they participate, for the trained sportmen, to a better gestion of the muscle glycogen storage : their utilization during exercise is decreased,

- they increase the lean body mass, with an increase of the protein synthesis and a decrease of the protein catabolism, leading also to an increase of the muscle force under training. There is no beneficial effect upon the tendons,

- they have an immunomodulation action.

Key-words : Androgens, physiology, muscular exercise, sports, beneficial effects. 